\title{
IL-18 polymorphisms contribute to hepatitis $B$ virus-related cirrhosis and hepatocellular carcinoma susceptibility in Chinese population: a case-control study
}

\author{
Zhi-Jun Dai, ${ }^{1, *}$ Xing-Han Liu ${ }^{1, *}$, Meng Wang ${ }^{1, *}$, Yan Guo ${ }^{2}$, Wenge Zhu ${ }^{3}$, Xiao $\mathrm{Li}^{4}$,

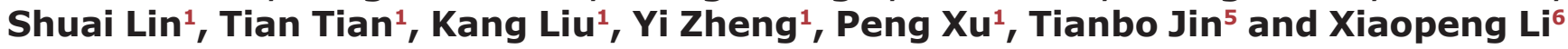 \\ ${ }^{1}$ Department of Oncology, The Second Affiliated Hospital of Xi'an Jiaotong University, Xi'an, China \\ ${ }^{2}$ School of Life Science and Technology, Xi'an Jiaotong University, Xi'an, China \\ ${ }^{3}$ Department of Biochemistry and Molecular Medicine, The George Washington University Medical School, Washington, DC, USA \\ ${ }^{4}$ Department of Hepatobiliary Surgery, Xijing Hospital, Fourth Military Medical University, Xi'an, China \\ ${ }^{5}$ National Engineering Research Center for Miniaturized Detection Systems, School of Life Sciences, Northwest University, \\ Xi'an, China \\ ${ }^{6}$ Department of Ultrasound, The Second Affiliated Hospital of Xi'an Jiaotong University, Xi'an, China \\ *These authors contributed equally to this work
}

Correspondence to: Xiaopeng Li, email: xiaopengli8@126.com

Zhi-Jun Dai, email: dzj0911@126.com

Keywords: $I L-18, H B V$, susceptibility

Received: February 24, 2017 Accepted: June 04, $2017 \quad$ Published: June 17, 2017

Copyright: Dai et al. This is an open-access article distributed under the terms of the Creative Commons Attribution License 3.0 (CC BY 3.0), which permits unrestricted use, distribution, and reproduction in any medium, provided the original author and source are credited.

\section{ABSTRACT}

IL-18 polymorphisms influence the transcriptional activity of the IL-18 gene and associated with various diseases. However, their relationships with hepatitis B virus-related liver diseases had not reached a consensus. So we conducted this casecontrol study with a view to clarifying the association. We included four groups: healthy controls, chronic hepatitis B virus (CHB) carriers, liver cirrhosis (LC) and hepatocellular carcinoma (HCC) groups with each group of 250 persons. Odd ratios (ORs) and $\mathbf{9 5 \%}$ confidence intervals $(95 \% \mathrm{CIs})$ with or without adjustment were calculated. Haplotype analysis was also performed. The results showed people carrying rs187238 CG genotype had a lower risk of LC (CG vs. CC: OR $=0.59,95 \%$ CI $=0.38-0.91, P=0.02$ ), while GG genotype carriers had a higher risk of HCC (GG vs. CC+CG: OR $=4.73,95 \% \mathrm{CI}=$ 1.01-22.1, $P=0.03$ ) than those with CC and CG genotypes in healthy group. Rs 187238 GG genotype increased the risk from CHB to LC status (GG vs. CC: OR $=4.81,95 \% \mathrm{CI}=$ 1.03-22.6; GG vs. CC+CG: $O R=4.73,95 \% C I=1.01-22.1)$, meanwhile the trend also existed by controlling confounding factors (GG vs. CC: $O R=6.25,95 \% \mathrm{CI}=1.09-35.8$; GG vs. CC+CG: $O R=5.91,95 \% C I=1.04-33.7)$. Haplotype Crs187238Trs 1946518 moderately decreased the risk of CHB carriers developing into HCC (OR $=0.69,95 \% \mathrm{CI}$ $=0.50-0.96, P=0.03$ ) after adjustment. In conclusion, IL-18 rs187238 GG genotype may increase the risk of HCC in healthy population and the risk of LC in CHB carriers.

\section{INTRODUCTION}

From 1990 to 2013, ranking of viral hepatitis on leading death causes worldwide rose from the tenth to seventh [1]. Despite the availability of an effective vaccine and antiviral approaches, more than 250 million people suffer from chronic hepatitis B (CHB) infection and nearly one million deaths occur each year owing to complications worldwide $[2,3]$. Genomic mutations including drugresistant mutants and vaccine-escape mutants (G145R, G145A, F134L and others) hinder the treatment and prevention of hepatitis B virus (HBV) infection [2]. 
Prospective studies revealed chronically infected HBV patients had an up to 100 -fold increased risk for progressing to hepatocellular carcinoma (HCC) [4]. Genomic mutations were significantly involved in the development of HBVrelated HCC. P203Q and S210R which are mutations in $\mathrm{HBsAg} \mathrm{C}$-terminus hamper HBsAg-secretion and correlate with increased cellular proliferation and $\mathrm{HBV}$-induced HCC [5]. A meta-analysis performed by Tian $\mathrm{T}$ et al. indicated that miR-146a C $>\mathrm{G}$ increased HBV-related HCC risk while miR-196a-2 $\mathrm{C}>\mathrm{T}$ decreased the risk of $\mathrm{HBV}$ related HCC, especially in the Chinese population [6]. A retrospective study found the $\mathrm{AG}$ genotype and $\mathrm{G}$ allele for $\mathrm{A} 3 \mathrm{G}$ rs8177832 polymorphism related to a decreased risk of CHB and HBV-related HCC and TT genotype of rs2011861 polymorphism confer to an increased risk of HBV-related HCC [7].

Interleukin-18 (IL-18), firstly described as interferon (IFN)-gamma inducing factor, is one of the members of the IL-1 cytokine superfamily [8, 9]. IL18 modulates the Th1 response together with IL-12 to produce IFN-gamma [10]. It has been shown that IL-18 can also influence Th2 response in synergy with other Th2-stimulating factors [11]. Thus, IL-18 is capable of increasing the activity of both Th1-type and Th2-type $\mathrm{CD}^{+} \mathrm{T}$ cells depending on its cytokine milieu [12]. CD ${ }^{+}$ $\mathrm{T}$ cells can contribute directly to disease pathogenesis and inhibit viral replication during HBV infection [13]. Cytotoxic $\mathrm{T}$ lymphocytes can directly recognize and kill infected hepatocytes [14]. IL-18 plays a part in the clearance of viruses, partly by the induction of cytotoxic $\mathrm{T}$ cells [15]. All the findings suggest IL-18 may be associated with HBV and HCC. Human IL-18 gene is located on chromosome 11q22.2-22.3 and contains six exons. Within the promoter region of the IL-18 gene, two polymorphisms (rs187238 and rs1946518) have been identified, which influence the transcriptional activity of the IL-18 gene [16]. Previous studies demonstrated an significant association between rs 1946518 polymorphism and various diseases risk, such as asthma, Crohn's disease and breast cancer [17-19]. Rs1946518 polymorphism was also suggested to be associated with hepatitis $\mathrm{C}$ virus (HCV) infections [20]. Hass SL et al. found though different genotypes of the two SNPs distributed equally in HCV patients and health controls, they were related to treatment response in hepatitis $\mathrm{C}$ patients [21]. Li Y et al. found no association between the two SNPs and HBV recurrence after liver transplantation in Chinese Han people [22].

Several researches have studied the impact of the two polymorphisms on HBV-induced liver diseases, but no consistent conclusions had been achieved [23-26]. The purpose of this study was to assess the relationship of rs 187238 and rs1946518 polymorphisms and the risk of HBV-liver cirrhosis (LC) and HBV-related HCC in the northwestern Chinese population.

\section{RESULTS}

\section{Characteristics of the study population}

The included subjects were equally categorized into four groups with 250 participants, healthy controls, CHB, CHB-positive LC and CHB-positive HCC (Table 1). The average ( \pm standard deviation) of the four parts were $55.71 \pm 9.17,54.17 \pm 10.37,53.12 \pm 10.58$ and $54.42 \pm$ 12.00 , respectively. Males occupied $80.4 \%, 72.8 \%, 70.8 \%$ and $77.6 \%$ in the healthy group, CHB carriers, LC and HCC patients, respectively. The four groups did differ significantly in alcohol history $(P=0.002)$ and diabetes history $(P=0.021)$, while they had no meaningful difference in smoking history $(P=0.100)$ and family history $(P=0.647)$. Furthermore, patients carrying CHB related liver diseases had higher laboratory parameters than healthy controls, including T-Bil, ALT and AST levels $(P<0.001)$, which are evidences of liver injury [27].

The alleles and genotype distributions of IL18 polymorphisms were shown in Table 2 . Genotype frequencies of the two polymorphisms in control group were consistent with HWE (Table 3, rs 187238: $P=0.142$; rs 1946518: $P=0.90)$. A LC patient and five HCC patients were failed to detect 187328 genotype, and three patients with HCC was failed to detect rs 1946518 polymorphism. For rs187238 polymorphism, as displayed in Table 2, healthy controls with $\mathrm{CC}, \mathrm{CG}$ and GG genotypes were 183, 65 and 2; CHB carriers were 200, 48, and 2; LC patients were 202, 42 and 5, and HCC subjects were 187, 49 and 9, respectively. For rs1946518 polymorphism, GG, GT and TT genotype accounts for $25.6 \%, 49.6 \%$ and $24.8 \%$ in disease free controls, $24.4 \%, 53.6 \%$ and $22 \%$ in CHB carriers, $28.8 \%, 47.2 \%$ and $24 \%$ in LC patients, and $26.8 \%, 47.2 \%$ and $24.8 \%$ in HCC patients, respectively (Table 2).

\section{Association between IL-18 polymorphisms and the risk of $L C$}

Compared with healthy people carrying rs187238 CC genotype, people with CG genotype had a low risk of LC (Table 4, CG vs. CC: $\mathrm{OR}=0.59,95 \% \mathrm{CI}=0.38-0.91$, $P=0.02)$. The genotype GG at 187238 was more frequent in LC patients than CHB patients ( $2 \%$ vs. $0.8 \%)$ and logistic regression analyses showed a higher LC risk in CHB carriers with rs 187238 GG genotype than individuals with $\mathrm{CG}$ or CC genotypes (GG vs. CC: $\mathrm{OR}=6.25,95 \% \mathrm{CI}$ $=1.09-35.8$; $\mathrm{GG}$ vs. $\mathrm{CC}+\mathrm{CG}$ : $\mathrm{OR}=5.91,95 \% \mathrm{CI}=$ 1.04-33.7). However, after controlling for gender, age, smoking and alcohol consumption, the result indicated no significant difference in rs 187238 polymorphism between LC and health controls (Table 5). No relationship between rs1946518 polymorphism and LC susceptibility was detected (Tables 4 and 5). 
Table 1: Characteristics of included subjects

\begin{tabular}{|c|c|c|c|c|c|}
\hline Characteristics & $\begin{array}{l}\text { Healthy } \\
\text { controls }\end{array}$ & CHB & $\begin{array}{c}\text { CHB-related } \\
\text { LC }\end{array}$ & CHB-related HCC & $P$ \\
\hline Total number & 250 & 250 & 250 & 250 & \\
\hline Age $($ mean $\pm \mathrm{SD})$ & $55.71 \pm 9.17$ & $54.17 \pm 10.37$ & $53.12 \pm 10.58$ & $54.47 \pm 12.00$ & 0.056 \\
\hline \multicolumn{6}{|l|}{ Gender } \\
\hline Male & 201 & 182 & 177 & 194 & 0.051 \\
\hline Female & 49 & 68 & 73 & 56 & \\
\hline \multicolumn{6}{|l|}{ Alcohol history } \\
\hline yes & 22 & 53 & 39 & 42 & 0.002 \\
\hline no & 228 & 197 & 211 & 208 & \\
\hline \multicolumn{6}{|l|}{ Smoking history } \\
\hline yes & 145 & 137 & 118 & 131 & 0.100 \\
\hline no & 105 & 113 & 132 & 119 & \\
\hline \multicolumn{6}{|l|}{ Diabetes history } \\
\hline Yes & 22 & 30 & 39 & 44 & 0.021 \\
\hline no & 228 & 220 & 211 & 206 & \\
\hline \multicolumn{6}{|l|}{ Family history } \\
\hline Yes & 8 & 7 & 12 & 10 & 0.647 \\
\hline No & 242 & 243 & 238 & 240 & \\
\hline \multicolumn{6}{|l|}{ Laboratory parameters } \\
\hline T-Bil level (umol/L) & $8.49 \pm 4.09$ & $14.35 \pm 5.29$ & $39.22 \pm 11.58$ & $36.76 \pm 10.11$ & $<0.001$ \\
\hline ALT (U/L) & $15.36 \pm 5.27$ & $65.33 \pm 13.40$ & $63.84 \pm 21.26$ & $71.03 \pm 16.50$ & $<0.001$ \\
\hline AST (U/L) & $12.35 \pm 4.05$ & $49.62 \pm 15.16$ & $47.99 \pm 24.83$ & $58.21 \pm 17.55$ & $<0.001$ \\
\hline $\operatorname{AFP}(\mathrm{ng} / \mathrm{ml})$ & No data & $7.94 \pm 3.31$ & $43.43 \pm 29.46$ & $1629.59 \pm 625.28$ & $<0.001$ \\
\hline $\begin{array}{l}\text { Number of patients with } \\
\text { AFP } \leq 400 \mathrm{ng} / \mathrm{ml}\end{array}$ & - & - & - & 156 & \\
\hline
\end{tabular}

CHB, chronic hepatitis B; LC, liver cirrhosis; HCC, hepatocellular carcinoma; AFP, alpha-fetoprotein.

\section{Association between IL-18 polymorphisms and the risk of $\mathrm{HCC}$}

A significant higher risk of $\mathrm{HCC}$ was associated with rs187238 GG genotype, when compared with healthy controls with CC/CG genotypes (Table 4, GG vs. CC+CG: $\mathrm{OR}=4.73$, $95 \% \mathrm{CI}=1.01-22.1)$ and the similar trend was also observed after adjustment for gender, age, smoking and alcohol consumption (Table 5, GG vs. CC: $\mathrm{OR}=6.96,95 \% \mathrm{CI}=$ $1.24-39.0 ; \mathrm{GG}$ vs. $\mathrm{CC}+\mathrm{CG}$ : $\mathrm{OR}=7.64,95 \% \mathrm{CI}=1.37-42.5)$. Unfortunately, whether compared with healthy group or CHB carriers, rs 1946518 polymorphism did not have a significant influence on developing to HCC (Tables 4 and 5).

\section{Association between IL-18 polymorphisms and the risk from $\mathrm{LC}$ to $\mathrm{HCC}$}

Displayed in Table 5, neither of the two polymorphisms hampered or accelerated patients from LC progressing to HCC (Table 4). Adjusting for gender, age, smoking and drinking status did not have a meaning change (Table 5).

\section{Haplotype distributions of IL-18 polymorphisms in healthy controls, HBV, LC and HCC patients}

We further performed the haplotype analyses to evaluate the haplotype frequencies of rs 187238 and rs1946518 polymorphisms. Three haplotypes $\left(\mathrm{G}_{\mathrm{rs} 187238} \mathrm{~T}_{\mathrm{rs} 1946518}, \mathrm{C}_{\mathrm{rs} 187238} \mathrm{~T}_{\mathrm{rs} 1946518}\right.$ and $\left.\mathrm{C}_{\mathrm{rs} 187238} \mathrm{G}_{\mathrm{rs} 1946518}\right)$ were analyzed. As shown in Table 6, the two polymorphisms had no interaction. However, after adjustment by gender, age, smoking and drinking, haplotype $\mathrm{C}_{\mathrm{rs} 187238} \mathrm{~T}_{\mathrm{rs} 1946518}$ moderately decreased the risk of CHB carriers developing into HCC (Table 7, OR $=0.69,95 \% \mathrm{CI}=0.50-0.96, \mathrm{P}=0.03$ ).

\section{DISCUSSION}

IL-18 promoter single-nucleotide polymorphisms (SNPs) were suggested to associate with different 
Table 2: Allele and genotype distributions of rs187238 and rs1946518 polymorphisms in healthy controls, CHB, LC and HCC patients

\begin{tabular}{|c|c|c|c|c|c|}
\hline Model & & Control $(n, \%)$ & $\mathrm{CHB}(n, \%)$ & $\mathrm{LC}(n, \%)$ & $\mathrm{HCC}(n, \%)$ \\
\hline \multicolumn{6}{|l|}{ rs 187238} \\
\hline \multirow[t]{2}{*}{ Allele } & $\mathrm{C}$ & $431(86.2 \%)$ & $448(89.6 \%)$ & $446(89.6 \%)$ & $423(86.3 \%)$ \\
\hline & G & $69(13.8 \%)$ & $52(10.4 \%)$ & $52(10.4 \%)$ & $67(13.7 \%)$ \\
\hline \multirow[t]{3}{*}{ Codominant model } & $\mathrm{CC}$ & $183(73.2 \%)$ & $200(80 \%)$ & $202(81.1 \%)$ & $187(76.3 \%)$ \\
\hline & $\mathrm{CG}$ & $65(26 \%)$ & $48(19.2 \%)$ & $42(16.9 \%)$ & $49(20 \%)$ \\
\hline & GG & $2(0.8 \%)$ & $2(0.8 \%)$ & $5(2 \%)$ & $9(3.7 \%)$ \\
\hline \multirow[t]{2}{*}{ Dominant model } & $\mathrm{CC}$ & $183(73.2 \%)$ & $200(80 \%)$ & $202(81.1 \%)$ & $187(76.3 \%)$ \\
\hline & $\mathrm{CG}+\mathrm{GG}$ & $67(26.8 \%)$ & $50(20 \%)$ & $47(18.9 \%)$ & $58(23.7 \%)$ \\
\hline \multirow[t]{2}{*}{ Recessive model } & $\mathrm{CC}+\mathrm{CG}$ & $248(99.2 \%)$ & $248(99.2 \%)$ & $244(98 \%)$ & $236(96.3 \%)$ \\
\hline & GG & $2(0.8 \%)$ & $2(0.8 \%)$ & $5(2 \%)$ & $9(3.7 \%)$ \\
\hline \multicolumn{6}{|l|}{ rs 1946518} \\
\hline \multirow[t]{2}{*}{ Allele } & $\mathrm{G}$ & $252(50.4 \%)$ & $256(51.2 \%)$ & $262(52.4 \%)$ & $252(51 \%)$ \\
\hline & $\mathrm{T}$ & $248(49.6 \%)$ & $244(48.8 \%)$ & $238(47.6 \%)$ & $242(49 \%)$ \\
\hline \multirow[t]{3}{*}{ Codominant model } & GG & $64(25.6 \%)$ & $61(24.4 \%)$ & $72(28.8 \%)$ & $67(27.1 \%)$ \\
\hline & GT & $124(49.6 \%)$ & $134(53.6 \%)$ & $118(47.2 \%)$ & $118(47.8 \%)$ \\
\hline & TT & $62(24.8 \%)$ & $55(22 \%)$ & $60(24 \%)$ & $62(25.1 \%)$ \\
\hline \multirow[t]{2}{*}{ Dominant model } & GG & $64(25.6 \%)$ & $61(24.4)$ & $72(28.8 \%)$ & $67(27.1 \%)$ \\
\hline & $\mathrm{GT}+\mathrm{TT}$ & $186(74.4 \%)$ & $189(75.6 \%)$ & $178(71.2 \%)$ & $180(72.9 \%)$ \\
\hline \multirow[t]{2}{*}{ Recessive model } & $\mathrm{GG}+\mathrm{GT}$ & $188(75.2 \%)$ & $195(78 \%)$ & $190(76 \%)$ & $185(74.9 \%)$ \\
\hline & TT & $62(24.8 \%)$ & $55(22 \%)$ & $60(24 \%)$ & $62(25.1 \%)$ \\
\hline \multirow[t]{3}{*}{ Haplotype } & GT & $13.80 \%$ & $10.40 \%$ & $10.44 \%$ & $13.52 \%$ \\
\hline & $\mathrm{CT}$ & $35.80 \%$ & $38.40 \%$ & $36.95 \%$ & $35.66 \%$ \\
\hline & $\mathrm{CG}$ & $50.40 \%$ & $51.20 \%$ & $52.61 \%$ & $50.82 \%$ \\
\hline
\end{tabular}

$\mathrm{HWE}=$ hardy-weinberg equilibrium, $\mathrm{CHB}=$ chronic hepatitis $\mathrm{B}, \mathrm{LC}=$ liver cirrhosis, $\mathrm{HCC}=$ hepatocellular carcinoma, $\mathrm{GT}$, $\mathrm{G}_{\mathrm{rs} 187238} \mathrm{~T}_{\mathrm{rs} 1946518} ; \mathrm{CT}, \mathrm{C}_{\mathrm{rs} 187238} \mathrm{~T}_{\mathrm{rs} 1946518} ; \mathrm{CG}, \mathrm{C}_{\mathrm{rs} 187238} \mathrm{G}_{\mathrm{rs} 1946518}$.

Table 3: Hardy-Weinberg equilibrium test for the rs187238 and rs1946518 polymorphisms in the control group

\begin{tabular}{lccc}
\hline & Observed frequency & Expected frequency & P \\
\hline Rs187238 & 183 & 185.761 & 0.142 \\
CC & 65 & 59.478 \\
CG & 2 & 4.761 & \\
GG & & & 0.900 \\
Rs1946518 & 64 & 63.504 & 124.992 \\
GG & 124 & 61.504 & \\
GT & 62 & & \\
TT & & & \\
\hline
\end{tabular}

expression of IL-18 by causing differences in transcription factor binding [28]. Rs187238 polymorphism, which has a change from $\mathrm{G}$ to $\mathrm{C}$ changes the H4TF-1 nuclear factor binding site, while rs1946518 polymorphism disrupts a potential cAMP-responsive element binding protein binding site [16]. Furthermore, rs187238 polymorphism could affect significantly IL-18 transcriptional activity [29]. Rs 1946518 polymorphism is highly linked with the pathogenesis of chronic $\mathrm{C}$ virus infection (HCV) and HCVinfected patients had higher levels of IL-18 that correlate 
Table 4: Association between IL-18 polymorphisms and LC and HCC risk

\begin{tabular}{|c|c|c|c|c|c|c|c|c|c|c|}
\hline \multirow{2}{*}{$\begin{array}{c}\text { Model } \\
\text { Rs187238 }\end{array}$} & \multicolumn{2}{|c|}{ LC vs. Controls } & \multicolumn{2}{|c|}{ HCC vs. Controls } & \multicolumn{2}{|c|}{ LC vs. CHB } & \multicolumn{2}{|c|}{ HCC vs. CHB } & \multicolumn{2}{|c|}{ HCC vs. LC } \\
\hline & $\begin{array}{c}\text { OR } \\
(95 \% \mathrm{CI})\end{array}$ & $P$ & $\begin{array}{c}\text { OR } \\
(95 \% \mathrm{CI})\end{array}$ & $P$ & $\begin{array}{c}\text { OR } \\
(95 \% \mathrm{CI})\end{array}$ & $P$ & $\begin{array}{c}\text { OR } \\
(95 \% \mathrm{CI})\end{array}$ & $P$ & $\begin{array}{c}\text { OR } \\
(95 \% \mathrm{CI})\end{array}$ & $P$ \\
\hline \multicolumn{11}{|l|}{ Allele } \\
\hline $\mathrm{C}$ & $1^{\text {ref }}$ & 0.10 & $1^{\text {ref }}$ & 0.95 & $1^{\text {ref }}$ & 0.11 & $1^{\text {ref }}$ & 0.98 & $1^{\text {ref }}$ & 0.12 \\
\hline G & $\begin{array}{c}0.73 \\
(0.50-1.07)\end{array}$ & & $\begin{array}{c}0.99 \\
(0.69-1.42)\end{array}$ & & $\begin{array}{c}1.37 \\
(0.93-2.01)\end{array}$ & & $\begin{array}{c}1.004 \\
(0.67-1.51)\end{array}$ & & $\begin{array}{c}1.36 \\
(0.92-2.00)\end{array}$ & \\
\hline \multicolumn{11}{|c|}{ Codominant model } \\
\hline $\mathrm{CC}$ & $1^{\text {ref }}$ & & $1^{\text {ref }}$ & & $1^{\text {ref }}$ & & $1^{\text {ref }}$ & & $1^{\text {ref }}$ & \\
\hline $\mathrm{CG}$ & $\begin{array}{c}0.59 \\
(0.38-0.91)\end{array}$ & 0.02 & $\begin{array}{c}0.74 \\
(0.48-1.13)\end{array}$ & 0.16 & $\begin{array}{c}1.09 \\
(0.70-1.70)\end{array}$ & 0.70 & $\begin{array}{c}0.87 \\
(0.55-1.37)\end{array}$ & 0.54 & $\begin{array}{c}1.26 \\
(0.80-2.00)\end{array}$ & 0.32 \\
\hline GG & $\begin{array}{c}2.26 \\
(0.43-11.8)\end{array}$ & 0.54 & $\begin{array}{c}4.40 \\
(0.94-20.7)\end{array}$ & 0.04 & $\begin{array}{c}4.81 \\
(1.03-22.6)\end{array}$ & 0.03 & $\begin{array}{c}2.28 \\
(0.47-12.91)\end{array}$ & 0.47 & $\begin{array}{c}1.94 \\
(0.64-5.91)\end{array}$ & 0.23 \\
\hline \multicolumn{11}{|c|}{ Dominant model } \\
\hline $\mathrm{CC}$ & $1^{\text {ref }}$ & 0.04 & $1^{\text {ref }}$ & 0.02 & $1^{\text {ref }}$ & 0.32 & $1^{\text {ref }}$ & 0.75 & $1^{\mathrm{ref}}$ & 0.19 \\
\hline $\mathrm{CG}+\mathrm{GG}$ & $\begin{array}{c}0.64 \\
(0.42-0.97)\end{array}$ & & $\begin{array}{c}0.85 \\
(0.56-1.27)\end{array}$ & & $\begin{array}{c}1.24 \\
(0.81-1.90)\end{array}$ & & $\begin{array}{c}0.93 \\
(0.60-1.45)\end{array}$ & & $\begin{array}{c}1.33 \\
(0.86-2.06)\end{array}$ & \\
\hline \multicolumn{11}{|c|}{ Recessive model } \\
\hline $\mathrm{CC}+\mathrm{CG}$ & $1^{\text {ref }}$ & 0.44 & $1^{\text {ref }}$ & 0.03 & $1^{\text {ref }}$ & 0.03 & $1^{\text {ref }}$ & 0.44 & $1^{\text {ref }}$ & 0.26 \\
\hline GG & $\begin{array}{c}2.54 \\
(0.49-13.2)\end{array}$ & & $\begin{array}{c}4.73 \\
(1.01-22.1)\end{array}$ & & $\begin{array}{c}4.73 \\
(1.01-22.1)\end{array}$ & & $\begin{array}{c}2.54 \\
(0.49-13.2)\end{array}$ & & $\begin{array}{c}1.86 \\
(0.61-5.63)\end{array}$ & \\
\hline Rs1946518 & $\begin{array}{c}\text { OR } \\
(95 \% \mathrm{CI})\end{array}$ & $\mathrm{P}$ & $\begin{array}{c}\text { OR } \\
(95 \% \mathrm{CI})\end{array}$ & $\mathrm{P}$ & $\begin{array}{c}\text { OR } \\
(95 \% \mathrm{CI})\end{array}$ & $P$ & $\begin{array}{c}\text { OR } \\
(95 \% \mathrm{CI})\end{array}$ & $\mathrm{P}$ & $\begin{array}{c}\text { OR } \\
(95 \% \mathrm{CI})\end{array}$ & $\mathrm{P}$ \\
\hline \multicolumn{11}{|l|}{ Allele } \\
\hline G & $1^{\text {ref }}$ & 0.53 & $1^{\text {ref }}$ & 0.85 & $1^{\text {ref }}$ & 0.95 & $1^{\text {ref }}$ & 0.70 & $1^{\text {ref }}$ & 0.66 \\
\hline $\mathrm{T}$ & $\begin{array}{c}0.92 \\
(0.72-1.18)\end{array}$ & & $\begin{array}{c}0.98 \\
(0.76-1.25)\end{array}$ & & $\begin{array}{c}1.01 \\
(0.79-1.29)\end{array}$ & & $\begin{array}{c}0.95 \\
(0.74-1.22)\end{array}$ & & $\begin{array}{c}1.06 \\
(0.82-1.36)\end{array}$ & \\
\hline \multicolumn{11}{|c|}{ Codominant model } \\
\hline GG & $1^{\text {ref }}$ & & $1^{\text {ref }}$ & & $1^{\text {ref }}$ & & $1^{\text {ref }}$ & & $1^{\text {ref }}$ & \\
\hline GT & $\begin{array}{c}0.85 \\
(0.56-1.29)\end{array}$ & 0.44 & $\begin{array}{c}0.91 \\
(0.59-1.39)\end{array}$ & 0.66 & $\begin{array}{c}0.80 \\
(0.52-1.23)\end{array}$ & 0.31 & $\begin{array}{c}0.75 \\
(0.49-1.14)\end{array}$ & 0.17 & $\begin{array}{c}0.92 \\
(0.60-1.42)\end{array}$ & 0.72 \\
\hline TT & $\begin{array}{c}0.86 \\
(0.53-1.40)\end{array}$ & 0.55 & $\begin{array}{c}0.96 \\
(0.58-1.56)\end{array}$ & 0.86 & $\begin{array}{c}1.03 \\
(0.62-1.70)\end{array}$ & 0.92 & $\begin{array}{c}0.92 \\
(0.56-1.52)\end{array}$ & 0.76 & $\begin{array}{c}1.11 \\
(0.68-1.81)\end{array}$ & 0.67 \\
\hline \multicolumn{11}{|c|}{ Dominant model } \\
\hline GG & $1^{\text {ref }}$ & 0.42 & $1^{\text {ref }}$ & 0.70 & $1^{\text {ref }}$ & 0.49 & $1^{\text {ref }}$ & 0.27 & $1^{\mathrm{ref}}$ & 0.68 \\
\hline $\mathrm{GT}+\mathrm{TT}$ & $\begin{array}{c}0.85 \\
(0.57-1.26)\end{array}$ & & $\begin{array}{c}0.92 \\
(0.62-1.38)\end{array}$ & & $\begin{array}{c}0.87 \\
(0.58-1.30)\end{array}$ & & $\begin{array}{c}0.80 \\
(0.54-1.19)\end{array}$ & & $\begin{array}{c}1.09 \\
(0.73-1.61)\end{array}$ & \\
\hline \multicolumn{11}{|c|}{ Recessive model } \\
\hline $\mathrm{GG}+\mathrm{GT}$ & $1^{\text {ref }}$ & 0.84 & $1^{\text {ref }}$ & 0.94 & $1^{\text {ref }}$ & 0.42 & $1^{\text {ref }}$ & 0.60 & $1^{\text {ref }}$ & 0.78 \\
\hline TT & $\begin{array}{c}0.96 \\
(0.64-1.44)\end{array}$ & & $\begin{array}{c}1.02 \\
(0.68-1.53)\end{array}$ & & $\begin{array}{c}1.19 \\
(0.78-1.80)\end{array}$ & & $\begin{array}{c}1.12 \\
(0.74-1.70)\end{array}$ & & $\begin{array}{c}1.06 \\
(0.71-1.60)\end{array}$ & \\
\hline
\end{tabular}

CHB, chronic hepatitis B; LC, liver cirrhosis; HCC, hepatocellular carcinoma; OR, odd ratio; 95\%CI, 95\% confidence interval.

with disease severity [30]. The two polymorphisms were also related to susceptibility to various diseases, such as tuberculosis, type 1 diabetes, and CHB [31-33].

Recently, the association between the two polymorphisms (rs187238 and rs1946518 polymorphisms) and HBV-related liver diseases were frequently researched. Lau HK et al. included 559 healthy controls and 342 HCC patients to investigate the relationship and finally found rs $187238 \mathrm{GC}$ and CC genotypes not only increased the risk of HCC, but also was responsible for vascular invasion and enhanced the prognosis in HBV-related HCC [34]. Rs1946518 polymorphism was associated with different outcomes of HBV infection and spontaneous clearance [35]. On the contrary, a retrospective research in Japan enrolled $204 \mathrm{HBV}$-infected cases from 1999 to 2003, of those 161 people developed to chronic progressive liver 
Table 5: Association between IL-18 polymorphisms and LC and HCC risk adjusted by gender, age, smoking and drinking

\begin{tabular}{|c|c|c|c|c|c|c|c|c|c|c|c|c|}
\hline \multirow{2}{*}{$\begin{array}{c}\text { Model } \\
\text { Rs187238 }\end{array}$} & \multicolumn{2}{|c|}{ LC vs. Controls } & \multicolumn{3}{|c|}{ HCC vs. Controls } & \multicolumn{3}{|c|}{ LC vs. CHB } & \multicolumn{2}{|c|}{ HCC vs. CHB } & \multicolumn{2}{|c|}{ HCC vs. LC } \\
\hline & $\begin{array}{c}\mathrm{OR}^{*} \\
\left(95 \% \mathrm{CI}^{*}\right.\end{array}$ & $P^{*}$ & $\begin{array}{c}\mathrm{OR}^{*} \\
\left(95 \% \mathrm{CI}^{*}\right)\end{array}$ & & $P *$ & $\begin{array}{c}\text { OR }^{*} \\
\left(95 \% \mathrm{CI}^{*}\right)\end{array}$ & & $P^{*}$ & $\begin{array}{c}\mathrm{OR}^{*} \\
\left(95 \% \mathrm{CI}^{*}\right)\end{array}$ & $P^{*}$ & $\begin{array}{c}\mathrm{OR}^{*} \\
\left(95 \% \mathrm{CI}^{*}\right)\end{array}$ & $P *$ \\
\hline \multicolumn{13}{|l|}{ Allele } \\
\hline $\mathrm{C}$ & $1^{\text {ref }}$ & 0.51 & $1^{\text {ref }}$ & 0.82 & & $1^{\text {ref }}$ & 0.04 & $1^{\text {ref }}$ & & 0.78 & $1^{\text {ref }}$ & 0.23 \\
\hline G & $\begin{array}{l}0.85 \\
(0.51-1.39)\end{array}$ & & $\begin{array}{l}1.05 \\
(0.68-1.62)\end{array}$ & & & $\begin{array}{l}1.60 \\
(1.01-2.53)\end{array}$ & & $\begin{array}{l}1.06 \\
(0.69-1.65)\end{array}$ & & & $\begin{array}{l}1.29 \\
(0.86-1.93)\end{array}$ & \\
\hline \multicolumn{13}{|c|}{ Codominant model } \\
\hline $\mathrm{CC}$ & $1^{\text {ref }}$ & & $1^{\mathrm{ref}}$ & & & $1^{\text {ref }}$ & & & $1^{\text {ref }}$ & & $1^{\text {ref }}$ & \\
\hline CG & $\begin{array}{l}0.74 \\
(0.43-1.28)\end{array}$ & 0.28 & $\begin{array}{l}0.70 \\
(0.41-1.18)\end{array}$ & 0.18 & & $\begin{array}{l}1.31 \\
(0.76-2.27)\end{array}$ & 0.33 & & $\begin{array}{l}0.98 \\
(0.60-1.60)\end{array}$ & 0.92 & $\begin{array}{l}1.16 \\
(0.70-1.90)\end{array}$ & 0.57 \\
\hline GG & $\begin{array}{l}2.25 \\
(0.27-18.47)\end{array}$ & 0.45 & $\begin{array}{l}6.96 \\
(1.24-39.0)\end{array}$ & 0.03 & & $\begin{array}{l}6.25 \\
(1.09-35.8)\end{array}$ & 0.04 & & $\begin{array}{l}2.09 \\
(0.34- \\
12.82)\end{array}$ & 0.42 & $\begin{array}{l}2.38 \\
(0.62-9.10)\end{array}$ & 0.20 \\
\hline \multicolumn{13}{|l|}{ Dominant model } \\
\hline $\mathrm{CC}$ & $1^{\text {ref }}$ & 0.37 & $1^{\text {ref }}$ & 0.52 & $1^{\mathrm{ref}}$ & & 0.12 & & $1^{\text {ref }}$ & 0.94 & $1^{\text {ref }}$ & 0.35 \\
\hline $\mathrm{CG}+\mathrm{GG}$ & $\begin{array}{l}0.78 \\
(0.46-1.33)\end{array}$ & & $\begin{array}{l}0.85 \\
(0.52-1.40)\end{array}$ & & $\begin{array}{l}1.52 \\
(0.90-2.57)\end{array}$ & & & & $\begin{array}{l}1.02 \\
(0.63-1.65)\end{array}$ & & $\begin{array}{l}1.26 \\
(0.78-2.02)\end{array}$ & \\
\hline \multicolumn{13}{|l|}{ Recessive model } \\
\hline $\mathrm{CC}+\mathrm{CG}$ & $1^{\text {ref }}$ & 0.41 & $1^{\text {ref }}$ & 0.02 & $1^{\text {ref }}$ & & 0.046 & $1^{\text {ref }}$ & & 0.42 & $1^{\text {ref }}$ & 0.22 \\
\hline GG & $\begin{array}{l}2.41 \\
(0.29-19.82)\end{array}$ & & $\begin{array}{l}7.64 \\
(1.37-42.5)\end{array}$ & & $\begin{array}{l}5.91 \\
(1.04-33.7)\end{array}$ & & & $\begin{array}{l}2.10 \\
(0.34-12.85)\end{array}$ & & & $\begin{array}{l}2.32 \\
(0.61-8.81)\end{array}$ & \\
\hline Rs1946518 & $\begin{array}{l}\mathrm{OR}^{*} \\
\left(95 \% \mathrm{CI}^{*}\right)\end{array}$ & $\mathrm{P}^{*}$ & $\begin{array}{l}\text { OR* } \\
\left(95 \% \mathrm{CI}^{*}\right)\end{array}$ & $\mathrm{P}^{*}$ & $\begin{array}{l}\text { OR* } \\
\left(95 \% \mathrm{CI}^{*}\right)\end{array}$ & $\mathrm{P}^{*}$ & & $\begin{array}{l}\text { OR* } \\
\left(95 \% \mathrm{CI}^{*}\right)\end{array}$ & $\mathrm{P}^{*}$ & & $\begin{array}{l}\text { OR* } \\
\left(95 \% \mathrm{CI}^{*}\right)\end{array}$ & $\mathrm{P}^{*}$ \\
\hline \multicolumn{13}{|l|}{ Allele } \\
\hline G & $1^{\text {ref }}$ & 0.73 & $1^{\text {ref }}$ & 0.56 & $1^{\text {ref }}$ & 0.37 & & $1^{\text {ref }}$ & 0.72 & & $1^{\text {ref }}$ & 0.78 \\
\hline $\mathrm{T}$ & $\begin{array}{l}1.06 \\
(0.78-1.44)\end{array}$ & & $\begin{array}{l}0.91 \\
(0.67-1.24)\end{array}$ & & $\begin{array}{l}0.87 \\
(0.63-1.18)\end{array}$ & & & $\begin{array}{l}0.95 \\
(0.72-1.25)\end{array}$ & & & $\begin{array}{l}1.04 \\
(0.78-1.36)\end{array}$ & \\
\hline \multicolumn{13}{|c|}{ Codominant model } \\
\hline GG & $1^{\text {ref }}$ & & $1^{\text {ref }}$ & & $1^{\text {ref }}$ & & & $1^{\text {ref }}$ & & & $1^{\text {ref }}$ & \\
\hline GT & $\begin{array}{l}0.95 \\
(0.55-1.62)\end{array}$ & 0.84 & $\begin{array}{l}0.75 \\
(0.44-1.28)\end{array}$ & 0.29 & $\begin{array}{l}0.59 \\
(0.35-1.01)\end{array}$ & 0.05 & & $\begin{array}{l}0.72 \\
(0.45-1.15)\end{array}$ & 0.17 & & $\begin{array}{l}1.15 \\
(0.72-1.84)\end{array}$ & 0.55 \\
\hline TT & $\begin{array}{l}1.12 \\
(0.60-2.07)\end{array}$ & 0.72 & $\begin{array}{l}0.84 \\
(0.45-1.54)\end{array}$ & 0.57 & $\begin{array}{l}0.76 \\
(0.41-1.42)\end{array}$ & 0.39 & & $\begin{array}{l}0.92 \\
(0.53-1.58)\end{array}$ & 0.76 & & $\begin{array}{l}1.07 \\
(0.63-1.83)\end{array}$ & 0.80 \\
\hline \multicolumn{13}{|l|}{ Dominant model } \\
\hline GG & $1^{\text {ref }}$ & 0.99 & $1^{\text {ref }}$ & 0.32 & $1^{\mathrm{ref}}$ & 0.09 & & $1^{\text {ref }}$ & 0.27 & & $1^{\text {ref }}$ & 0.60 \\
\hline $\mathrm{GT}+\mathrm{TT}$ & $\begin{array}{l}1.002 \\
(0.61-1.66)\end{array}$ & & $\begin{array}{l}0.78 \\
(0.47-1.28)\end{array}$ & & $\begin{array}{l}0.64 \\
(0.39-1.06)\end{array}$ & & $\begin{array}{l}0.78 \\
(0.50-\end{array}$ & & & & $\begin{array}{l}1.12 \\
(0.73-1.73)\end{array}$ & \\
\hline \multicolumn{13}{|l|}{ Recessive model } \\
\hline $\mathrm{GG}+\mathrm{GT}$ & $1^{\text {ref }}$ & 0.57 & $1^{\text {ref }}$ & 0.97 & $1^{\mathrm{ref}}$ & 0.79 & & $1^{\text {ref }}$ & 0.58 & & $1^{\text {ref }}$ & 0.93 \\
\hline $\mathrm{TT}$ & $\begin{array}{l}1.16 \\
(0.70-1.93)\end{array}$ & & $\begin{array}{l}1.01 \\
(0.61-1.67)\end{array}$ & & $\begin{array}{l}1.07 \\
(0.64-1.80)\end{array}$ & & $\begin{array}{l}1.13 \\
(0.72-\end{array}$ & & & & $\begin{array}{l}0.98 \\
(0.63-1.54)\end{array}$ & \\
\hline
\end{tabular}

CHB, chronic hepatitis B; LC, liver cirrhosis; HCC, hepatocellular carcinoma; OR, odd ratio; $95 \% \mathrm{CI}, 95 \%$ confidence interval; OR*, OR values adjusted by gender, age, smoking and drinking; $\mathrm{P}^{*}, P$ values adjusted by gender, age, smoking and drinking.

disease with 62 CHB patients, 52 LC patients, and 47 LCC patients [36]. A protect effect of -607AA genotype and $-137 \mathrm{C}$ allele on the disease progression of HBV carriers was observed [36]. Rs 187238C allele was related to HCC in codominant and dominant models and its $\mathrm{C}$ allele showed significantly lower promoter activities which may affect IL-18 production and further affect CHB progression
[37]. However, several studies suggested reversed conclusions. Bao J et al. revealed rs 187238 not rs 1946518 polymorphism may be a protective factor against HCC [38]. The genotype and allele frequencies of rs 1946518 polymorphism showed no significant differences between any two groups of healthy controls, $\mathrm{CHB}$, and HCC groups [25]. A meta-analysis published in 2016, including eight 
Table 6: Analysis of IL-18 haplotype frequencies with the risk of LC and HCC

\begin{tabular}{lcccccccccc}
\hline & HCC vs. Controls & \multicolumn{2}{c}{ HCC vs. LC } & \multicolumn{2}{c}{ HCC vs. CHB } & \multicolumn{2}{c}{ LC vs. Controls } & \multicolumn{2}{c}{ LC vs. CHB } \\
\hline Haplotypes & $\begin{array}{c}\text { OR } \\
(\mathbf{9 5 \%} \mathbf{~ C I})\end{array}$ & $\boldsymbol{P}$ & $\begin{array}{c}\text { OR } \\
\mathbf{( 9 5 \%} \mathbf{~ C I})\end{array}$ & $\boldsymbol{P}$ & $\begin{array}{c}\text { OR } \\
\mathbf{9 5 \%} \mathbf{C I})\end{array}$ & $\boldsymbol{P}$ & $\begin{array}{c}\text { OR } \\
\mathbf{( 9 5 \% ~ C I ) ~}\end{array}$ & $\boldsymbol{P}$ & $\begin{array}{c}\text { OR } \\
\mathbf{( 9 5 \% ~ C I ) ~}\end{array}$ & $\boldsymbol{P}$ \\
\hline GT & 0.98 & 0.90 & 1.30 & 0.16 & 1.32 & 0.15 & 0.73 & 0.10 & 1.004 & 0.98 \\
& $(0.68-1.40)$ & & $(0.90-1.87)$ & & $(0.91-1.92)$ & & $(0.49-1.07)$ & & $(0.67-1.50)$ & \\
CT & 0.99 & 0.96 & 0.94 & 0.67 & 0.88 & 0.36 & 1.05 & 0.71 & 0.94 & 0.63 \\
& $(0.77-1.29)$ & & $(0.73-1.23)$ & & $(0.68-1.15)$ & & $(0.81-1.36)$ & & $(0.72-1.22)$ & \\
CG & 1.02 & 0.90 & 0.93 & 0.58 & 0.98 & 0.90 & 1.09 & 0.49 & 1.06 & 0.65 \\
& $(0.79-1.30)$ & & $(0.73-1.19)$ & & $(0.77-1.27)$ & & $(0.85-1.39)$ & & $(0.82-1.36)$ & \\
\hline
\end{tabular}

CHB, chronic hepatitis B; LC, liver cirrhosis; HCC, hepatocellular carcinoma; GT, $\mathrm{G}_{\mathrm{rs} 187238} \mathrm{~T}_{\mathrm{rs} 1946518} ; \mathrm{CT}, \mathrm{C}_{\mathrm{rs} 187238} \mathrm{~T}_{\mathrm{rs} 1946518} ; \mathrm{CG}$, $\mathrm{C}_{\mathrm{rs} 187238} \mathrm{G}_{\mathrm{rs} 1946518} ; \mathrm{OR}$, odd ratio; $95 \% \mathrm{CI}, 95 \%$ confidence interval.

Table 7: Analysis of IL-18 haplotype frequencies with the risk of LC and HCC adjusted by gender, age, smoking and drinking

\begin{tabular}{|c|c|c|c|c|c|c|c|c|c|c|}
\hline \multirow[b]{2}{*}{ Haplotypes } & \multicolumn{2}{|c|}{$\begin{array}{l}\text { HCC vs. } \\
\text { Controls } \\
\end{array}$} & \multicolumn{2}{|c|}{ HCC vs. LC } & \multicolumn{2}{|c|}{ HCC vs. CHB } & \multicolumn{2}{|c|}{ LC vs. Controls } & \multicolumn{2}{|c|}{ LC vs. CHB } \\
\hline & $\begin{array}{c}\text { OR* }^{*} \\
\left(95 \% \mathrm{CI}^{*}\right)\end{array}$ & $P^{*}$ & $\begin{array}{c}\mathrm{OR}^{*} \\
\left(95 \% \mathrm{CI}^{*}\right)\end{array}$ & $P^{*}$ & $\begin{array}{c}\text { OR* }^{*} \\
\left(95 \% \mathrm{CI}^{*}\right)\end{array}$ & $P^{*}$ & $\begin{array}{c}\text { OR* }^{*} \\
\left(95 \% \mathrm{CI}^{*}\right)\end{array}$ & $P^{*}$ & $\begin{array}{c}\mathrm{OR}^{*} \\
\left(95 \% \mathrm{CI}^{*}\right)\end{array}$ & $P^{*}$ \\
\hline GT & $\begin{array}{c}1.01 \\
(0.65-1.57)\end{array}$ & 0.96 & $\begin{array}{c}1.28 \\
(0.85-1.91)\end{array}$ & 0.24 & $\begin{array}{c}1.55 \\
(0.98-2.46)\end{array}$ & 0.06 & $\begin{array}{c}0.85 \\
(0.51-1.39)\end{array}$ & 0.51 & $\begin{array}{c}1.06 \\
(0.69-1.65)\end{array}$ & 0.78 \\
\hline CT & $\begin{array}{c}0.91 \\
(0.66-1.25)\end{array}$ & 0.55 & $\begin{array}{c}0.93 \\
(0.70-1.24)\end{array}$ & 0.62 & $\begin{array}{c}0.69 \\
(0.50-0.96)\end{array}$ & 0.03 & $\begin{array}{c}1.14 \\
(0.83-1.56)\end{array}$ & 0.43 & $\begin{array}{c}0.92 \\
(0.69-1.23)\end{array}$ & 0.58 \\
\hline CG & $\begin{array}{c}1.09 \\
(0.80-1.48)\end{array}$ & 0.59 & $\begin{array}{c}0.95 \\
(0.73-1.25)\end{array}$ & 0.74 & $\begin{array}{c}1.14 \\
(0.83-1.56)\end{array}$ & 0.43 & $\begin{array}{c}0.95 \\
(0.70-1.29)\end{array}$ & 0.73 & $\begin{array}{c}1.05 \\
(0.80-1.38)\end{array}$ & 0.72 \\
\hline
\end{tabular}

CHB, chronic hepatitis B; LC, liver cirrhosis; HCC, hepatocellular carcinoma; GT, $\mathrm{G}_{\mathrm{rs} 187238} \mathrm{~T}_{\mathrm{rs} 1946518} ; \mathrm{CT}, \mathrm{C}_{\mathrm{rs} 187238} \mathrm{~T}_{\mathrm{rs} 1946518}$; CG, $\mathrm{C}_{\mathrm{rs} 187238} \mathrm{G}_{\mathrm{rs} 1946518}$; OR, odd ratio; 95\%CI, 95\% confidence interval.

studies about rs 187238 polymorphism and seven studies about rs1946518 polymorphism, provided an evidence that the two polymorphisms had no influence on the risk of HCC in general or specifically of HBV-positive HCC [26].

Different from the previous studies, we enrolled 1000 people who were at different stages from health to HCC. The four groups did differ significantly in alcohol history and diabetes history. For LC patients carrying HBV, heavy alcohol consumption is a independent increased risk of HCC [39]. Lin CW et al. found in LC patients, the incidence of HCC was higher in patients with HBV infection and alcohol history than those with HBV infection or alcohol history alone [40]. Acetaldehyde, the ethanol metabolite, has carcinogenic characteristics by DNA binding [41]. Drinking-induced chronic oxidative stress and cytokine production could lead to LC and HCC development in the presence of chronic inflammation [42]. Alcohol could also promote HCC occurrence by deregulating the level of retinoic acid and S-adenosyl-Lmethionine, and activing the Wnt/ $\beta$-catenin pathway [43]. Diabetes could alter the hepatic function and the structure of the hepatocytes in the diabetic rats model, possibly by increasing hepatic oxidative stress, attenuating antioxidant capacity and further impairing liver [44, 45]. Our results indicated rs187238 GG genotype increased the risk of HCC in healthy population and the risk of LC in CHB carriers, and rs 187238 CG genotype decreased the risk of LC in health population. Epidemiological studies had demonstrated several risk factors for liver cancer including tobacco exposure, alcohol consumption, diabetes mellitus, $\mathrm{HBV}$ and HCV infection [46]. Though there is a significant difference in diabetes history between healthy controls and cases, researchers had different opinions about the impact of rs187238 and rs 1946518 polymorphisms on diabetes $[32,47]$. So we controlled for age, gender, smoking and alcohol consumption to minimize any possible confounding of results by these factors and finally found health people with GG genotype still had a higher risk of HCC and CHB patients carrying GG genotype still had a higher risk of LC. But no association between rs 1946518 polymorphism and HCC or LC risk was identified.

Our results should be interpreted with caution because of several limitations. Firstly, though we recruited 1000 samples in this study, the sample size of each group was relative small which may restrict its detail subgroup analysis by the clinical index.. Secondly, considering we just controlled four factors (age, gender, smoking and alcohol consumption), other factors including 
Table 8: Primers used in this study

\begin{tabular}{clll}
\hline SNP_ID & \multicolumn{1}{c}{ 1st-PCRP } & \multicolumn{1}{c}{ 2nd-PCRP } & UEP_SEQ \\
\hline rs187238 & ACGTTGGATGGCAGA & ACGTTGGATGACAGAGCC & GACCCAACTTTTACGGAAGAAAA \\
& GGATACGAGTACTTC & CCAACTTTTACG & \\
\multirow{2}{*}{ rs1946518 } & ACGTTGGATGCTCTCCCC & ACGTTGGATGTATCAGAT & ACACGGATACCATCATTAGAATTTTAT \\
& AAGCTTACTTTC & GCAAGCCACACG & \\
\hline
\end{tabular}

environmental background, treatment protocols and living habits may cause some bias. Thirdly, all participants were all from Shaanxi Province, China, which may not stand for all the Chinese population. Lastly, the fundamental experiments should be further conducted to validate our results and explore the possible mechanism.

In conclusion, our research revealed that rs 187238 GG genotype increased the risk of HCC in healthy population and the risk of LC in CHB carriers. However, rs 1946518 polymorphism had no impact on CHB-related liver diseases. These results indicated that IL-18 rs 187238 polymorphism may be involved in the progression of CHB-related diseases in Northwest Chinese population.

\section{MATERIALS AND METHODS}

\section{Ethnics statement}

This study was approved by the Ethics Committee of the Second Affiliated Hospital of Xi'an Jiaotong University (Xi'an, China). The research protocol was completed according to the approved guidelines.

\section{Study population}

For the current analysis, we established a casecontrol study of 250 healthy controls, $250 \mathrm{CHB}$ carriers, $250 \mathrm{LC}$ patients and $250 \mathrm{HCC}$ patients. HBV infection was detected by ELISA method that requiring positivity of serum HBsAg and serum negativity for anti-HCV. LC patients and HCC patients were confirmed by pathological diagnosed. All the subjects were from the Second Affiliated Hospital of Xi' an Jiaotong University, and Xijing Hospital of Fourth Military Medical University, Xi'an, Shaanxi Province, China. Every participant was interviewed in-person including questions on age, demographics, ethnicity, height and weight, smoking and drinking habits, and medical history. Blood samples were collected after interview.

\section{Genotyping assay}

The samples were centrifugated and stored in $-80^{\circ} \mathrm{C}$ freezers for long-term storage. The genomic DNA was extracted and concentrated by the method described in our previous studies $[48,49]$. Two tag-SNPs (rs 187238 and rs 1946518) were selected in this study. SNP genotyping was performed by the Sequenom MassARRAY RS1000, and the primers were listed in Table 8. The data analyses were completed by Sequenom Type 4.0.

\section{Statistical analyses}

All the statistical analyses were completed using the SPSS software package (version 20.0; SPSS Inc., Chicago, IL, USA). HWE was examined by comparing expected and observed frequencies using Alrlquin 3.1 program (L. Excoffier, CMPG, University of Bern, Switzerland). The genotype frequencies of observed values were compared with expected values obtained from HWE theory $(\mathrm{p} 2+2 \mathrm{pq}+\mathrm{q} 2=1 ; \mathrm{p}$ is the frequency of the wild-type allele and $q$ is the frequency of the variant allele). Haplotype analysis was performed by PHASE v2.1 software. The calculation was performed by $\chi 2$ test and the degree of freedom was 1 in the cases and controls. The significant difference in allele and genotype frequencies between cases and controls was determined by Pearson's $\chi 2$ test. ORs and $95 \%$ CIs were calculated with and without adjustment for gender, age, smoking and drinking. We evaluated the risk in the dominant model ( $\mathrm{AA}+\mathrm{Aa} v s$. aa), the recessive model (aa $v s . \mathrm{Aa}+\mathrm{AA})$, and the allele model (a vs. A) respectively (A: the major allele, a: the minor allele). A two-sided $P$-value $<0.05$ was considered statistically significant in all the tests.

\section{ACKNOWLEDGMENTS AND FUNDING}

This study was supported by National Natural Science Foundation, People's Republic of China (No 81471670); China Postdoctoral Science Foundation (No 2014M560791); the Fundamental Research Funds for the Central Universities, People's Republic of China (No 2014qngz-04); the International Cooperative Project (No 2013KW-32-01), and Science and Technology Plan of Innovation Project, Shaanxi Province, People's Republic of China (No 2015KTCL03-06).

\section{CONFLICTS OF INTEREST}

None.

\section{REFERENCES}

1. Stanaway JD, Flaxman AD, Naghavi M, Fitzmaurice C, Vos T, Abubakar I, Abu-Raddad LJ, Assadi R, Bhala N, Cowie B, Forouzanfour MH, Groeger J, Mohd Hanafiah K, 
et al. The global burden of viral hepatitis from 1990 to 2013: findings from the Global Burden of Disease Study 2013. Lancet. 2016; 388:1081-8. doi: 10.1016/s01406736(16)30579-7.

2. Nannini P, Sokal EM. Hepatitis B: changing epidemiology and interventions. Arch Dis Child. 2016; 102:0. doi: 10.1136/archdischild-2016-312043.

3. Chen GF, Wang C, Lau G. Treatment of chronic hepatitis B infection-2017. Liver Int. 2017; 37:59-66. doi: 10.1111/ liv.13309.

4. Van Hees S, Michielsen P, Vanwolleghem T. Circulating predictive and diagnostic biomarkers for hepatitis $\mathrm{B}$ virusassociated hepatocellular carcinoma. World J Gastroenterol. 2016; 22:8271-82. doi: 10.3748/wjg.v22.i37.8271.

5. Salpini R, Surdo M, Warner N, Cortese MF, Colledge D, Soppe S, Bellocchi MC, Armenia D, Carioti L, Continenza F, Di Carlo D, Saccomandi P, Mirabelli C, et al. Novel HBsAg mutations correlate with hepatocellular carcinoma, hamper HBsAg secretion and promote cell proliferation in vitro. Oncotarget. 2017; 8:15704-15715. doi: 10.18632/oncotarget.14944.

6. Tian T, Wang M, Zhu W, Dai ZM, Lin S, Yang PT, Liu XH, Liu K, Zhu YY, Zheng Y, Liu M, Dai ZJ. MiR-146a and miR196a-2 polymorphisms are associated with hepatitis virusrelated hepatocellular cancer risk: a meta-analysis. Aging (Albany NY). 2017; 9:381-392. doi: 10.18632/aging.101160.

7. He XT, Xu HQ, Wang XM, He XS, Niu JQ, Gao PJ. Association between polymorphisms of the APOBEC3G gene and chronic hepatitis $B$ viral infection and hepatitis $B$ virus-related hepatocellular carcinoma. World J Gastroenterol. 2017; 23:232-41. doi: 10.3748/wjg.v23.i2.232.

8. Okamura H, Nagata K, Komatsu T, Tanimoto T, Nukata Y, Tanabe F, Akita K, Torigoe K, Okura T, Fukuda S, Kurimoto M. A novel costimulatory factor for gamma interferon induction found in the livers of mice causes endotoxic shock. Infect Immun. 1995; 63:3966-72.

9. Dinarello CA, Novick D, Kim S, Kaplanski G. Interleukin-18 and IL-18 binding protein. Front Immunol. 2013; 4: 289. doi: 10.3389/fimmu.2013.00289.

10. Yoshimoto T, Takeda K, Tanaka T, Ohkusu K, Kashiwamura S, Okamura H, Akira S, Nakanishi K. IL12 up-regulates IL-18 receptor expression on T cells, Th1 cells, and B cells: synergism with IL-18 for IFN-gamma production. J Immunol. 1998; 161:3400-7.

11. Nakanishi $K$, Yoshimoto $T$, Tsutsui $H$, Okamura $H$. Interleukin-18 regulates both Th1 and Th2 responses. Annu Rev Immunol. 2001; 19:423-74. doi: 10.1146/annurev. immunol.19.1.423.

12. Fujibayashi Y, Fujimori Y, Kasumoto I, Kai S, Hara H, Okamura H, Tsutsui H, Ogawa H, Nakanishi K. Interleukin-18 regulates $\mathrm{T}$ helper 1 or 2 immune responses of human cord blood CD4+ V alpha $24+\mathrm{V}$ beta $11+$ natural killer T cells. Int J Mol Med. 2007; 20:241-5.

13. Franco A, Guidotti LG, Hobbs MV, Pasquetto V, Chisari FV. Pathogenetic effector function of CD4-positive T helper 1 cells in hepatitis B virus transgenic mice. J Immunol. 1997; 159:2001-8.

14. Xia Y, Protzer U. Control of Hepatitis B Virus by Cytokines. Viruses. 2017; 9. doi: 10.3390/v9010018.

15. Nakanishi $K$, Yoshimoto $T$, Tsutsui $H$, Okamura $H$. Interleukin-18 is a unique cytokine that stimulates both Th1 and Th2 responses depending on its cytokine milieu. Cytokine Growth Factor Rev. 2001; 12:53-72.

16. Giedraitis V, He B, Huang WX, Hillert J. Cloning and mutation analysis of the human IL-18 promoter: a possible role of polymorphisms in expression regulation. $\mathrm{J}$ Neuroimmunol. 2001; 112:146-52.

17. Ma Y, Zhang B, Tang RK, Liu Y, Peng GG. Interleukin-18 promoter polymorphism and asthma risk: a meta-analysis. Mol Biol Rep. 2012; 39:1371-6. doi: 10.1007/s11033-0110871-6.

18. Back LK, Farias TD, da Cunha PA, Muniz YC, Ribeiro MC, Fernandes BL, Fernandes CK, de Souza IR. Functional polymorphisms of interleukin-18 gene and risk of breast cancer in a Brazilian population. Tissue Antigens. 2014; 84:229-33. doi: 10.1111/tan.12367.

19. Gao SJ, Zhang L, Lu W, Wang L, Chen L, Zhu Z, Zhu HH. Interleukin-18 genetic polymorphisms contribute differentially to the susceptibility to Crohn's disease. World J Gastroenterol. 2015; 21:8711-22. doi: 10.3748/wjg.v21. i28.8711.

20. Yang Y, Liu H. Association between interleukin-18 gene promoter $(-607 \mathrm{C} / \mathrm{A}$ and $-137 \mathrm{G} / \mathrm{C})$ polymorphisms and chronic hepatitis $\mathrm{C}$ virus infections: A meta-analysis. Meta Gene. 2015; 5:21-31. doi: 10.1016/j.mgene.2015.04.004.

21. Haas SL, Weiss C, Bugert P, Gundt J, Witt H, Singer $\mathrm{MV}$, Berg T, Bocker U. Interleukin 18 promoter variants $(-137 \mathrm{G}>\mathrm{C}$ and $-607 \mathrm{C}>\mathrm{A})$ in patients with chronic hepatitis $\mathrm{C}$ : association with treatment response. J Clin Immunol. 2009; 29:620-8. doi: 10.1007/s10875-009-9302-Z.

22. Li Y, Shi Y, Chen J, Cai B, Ying B, Wang L. Association of polymorphisms in interleukin-18 and interleukin-28B with hepatitis B recurrence after liver transplantation in Chinese Han population. Int J Immunogenet. 2012; 39:346-52. doi: 10.1111/j.1744-313X.2012.01097.x.

23. Ferreira Sda C, Chacha SG, Souza FF, Teixeira AC, Santana Rde C, Deghaide NH, Rodrigues S, Marano LA, MendesJunior CT, Zucoloto S, Donadi EA, Martinelli Ade L. IL-18, TNF, and IFN-gamma alleles and genotypes are associated with susceptibility to chronic hepatitis B infection and severity of liver injury. J Med Virol. 2015; 87:1689-96. doi: 10.1002/jmv.24225.

24. Lu Y, Bao JG, Deng Y, Rong CZ, Liu YQ, Huang XL, Song LY, Li S, Qin X. Role of IL-18 Gene Promoter Polymorphisms, Serum IL-18 Levels, and Risk of Hepatitis B Virus-related Liver Disease in the Guangxi Zhuang Population: a Retrospective Case-Control Study. Asian Pac J Cancer Prev. 2015; 16:6019-26.

25. Zhang QX, Yao YQ, Li SL, Long Q. Association between interleukin-18 gene polymorphisms and hepatocellular 
carcinoma caused by hepatitis B virus. [Article in Chinese]. Zhonghua Gan Zang Bing Za Zhi. 2016; 24:352-7. doi: 10.3760/cma.j.issn.1007-3418.2016.05.008.

26. Zhu SL, Zhao Y, Hu XY, Luo T, Chen ZS, Zhang Y, Yang SH, Zhou L, Li LQ. Genetic polymorphisms -137 (rs187238) and -607 (rs1946518) in the interleukin-18 promoter may not be associated with development of hepatocellular carcinoma. Sci Rep. 2016; 6: 39404. doi: 10.1038/srep39404.

27. Lawrence YA, Steiner JM. Laboratory Evaluation of the Liver. Vet Clin North Am Small Anim Pract. 2017; 47:53953. doi: 10.1016/j.cvsm.2016.11.005.

28. Al-Khateeb GM, Sater MS, Finan RR, Mustafa FE, Al-Busaidi AS, Al-Sulaiti MA, Almawi WY. Analysis of interleukin-18 promoter polymorphisms and changes in interleukin-18 serum levels underscores the involvement of interleukin-18 in recurrent spontaneous miscarriage. Fertil Steril. 2011; 96:921-6. doi: 10.1016/j. fertnstert.2011.06.079.

29. Liang XH, Cheung W, Heng CK, Wang DY. Reduced transcriptional activity in individuals with IL-18 gene variants detected from functional but not association study. Biochem Biophys Res Commun. 2005; 338:736-41. doi: 10.1016/j.bbrc.2005.10.012.

30. Bouzgarrou N, Hassen E, Schvoerer E, Stoll-Keller F, Bahri O, Gabbouj S, Cheikh I, Maamouri N, Mammi N, Saffar H, Trabelsi A, Triki H, Chouchane L. Association of interleukin-18 polymorphisms and plasma level with the outcome of chronic HCV infection. J Med Virol. 2008; 80:607-14. doi: 10.1002/jmv.21079.

31. Hang PA, Wu JM, Li Y, Yang XS. Relationship of interleukin-18 gene promoter polymorphisms with chronic hepatitis B in Chinese Han population. [Article in Chinese]. Zhonghua Yi Xue Yi Chuan Xue Za Zhi. 2005; 22:528-32.

32. Lee YH, Kim JH, Song GG. Interleukin-18 promoter -607 $\mathrm{C} / \mathrm{A}$ and $-137 \mathrm{G} / \mathrm{C}$ polymorphisms and susceptibility to type 1 diabetes: A meta-analysis. Hum Immunol. 2015; 76:53745. doi: 10.1016/j.humimm.2015.06.012.

33. Zhou C, Ouyang N, Li QH, Luo SX, He Q, Lei H, Liu Q. The $-137 \mathrm{G} / \mathrm{C}$ single nucleotide polymorphism in IL-18 gene promoter contributes to tuberculosis susceptibility in Chinese Han population. Infect Genet Evol. 2015; 36:37680. doi: 10.1016/j.meegid.2015.10.014.

34. Lau HK, Hsieh MJ, Yang SF, Wang HL, Kuo WH, Lee HL, Yeh CB. Association between Interleukin-18 Polymorphisms and Hepatocellular Carcinoma Occurrence and Clinical Progression. Int J Med Sci. 2016; 13:556-61. doi: 10.7150/ijms. 15853 .

35. Karra VK, Gumma PK, Chowdhury SJ, Ruttala R, Polipalli SK, Chakravarti A, Kar P. IL-18 polymorphisms in hepatitis B virus related liver disease. Cytokine. 2015; 73:277-82. doi: 10.1016/j.cyto.2015.02.015.

36. Migita K, Sawakami-Kobayashi K, Maeda Y, Nakao K, Kondoh S, Sugiura M, Kawasumi R, Segawa O, Tajima H,
Machida M, Nakamura M, Yano K, Abiru S, et al. Interleukin-18 promoter polymorphisms and the disease progression of Hepatitis B virus-related liver disease. Trans1 Res. 2009; 153:91-6. doi: 10.1016/j.trs1.2008.11.008.

37. Kim YS, Cheong JY, Cho SW, Lee KM, Hwang JC, Oh B, Kimm K, Lee JA, Park BL, Cheong HS, Shin HD, Kim JH. A functional SNP of the Interleukin-18 gene is associated with the presence of hepatocellular carcinoma in hepatitis B virus-infected patients. Dig Dis Sci. 2009; 54:2722-8. doi: 10.1007/s10620-009-0970-6.

38. Teixeira AC, Mendes CT Jr, Marano LA, Deghaide NH, Secaf M, Elias J Jr, Muglia V, Donadi EA, Martinelli AL. Alleles and genotypes of polymorphisms of IL-18, TNF-alpha and IFN-gamma are associated with a higher risk and severity of hepatocellular carcinoma (HCC) in Brazil. Hum Immunol. 2013; 74:1024-9. doi: 10.1016/j. humimm.2013.04.029.

39. Zhang Y, Peng L, Cao Y, Zeng Z, Wu Y, Shi H, Chen S, Guo J. Analysis of the risk factors of hepatocellular carcinoma in cirrhotic patients with chronic hepatitis B. [Article in Chinese]. Zhonghua Gan Zang Bing Za Zhi. 2015; 23:512-6. doi: 10.3760/cma.j.issn.1007-3418.2015.07.009.

40. Lin CW, Lin CC, Mo LR, Chang CY, Perng DS, Hsu CC, Lo GH, Chen YS, Yen YC, Hu JT, Yu ML, Lee PH, Lin JT, et al. Heavy alcohol consumption increases the incidence of hepatocellular carcinoma in hepatitis B virus-related cirrhosis. J Hepatol. 2013; 58:730-5. doi: 10.1016/j. jhep.2012.11.045.

41. Seitz HK, Simanowski UA, Garzon FT, Rideout JM, Peters TJ, Koch A, Berger MR, Einecke H, Maiwald M. Possible role of acetaldehyde in ethanol-related rectal cocarcinogenesis in the rat. Gastroenterology. 1990; 98:406-13.

42. Nault JC. Pathogenesis of hepatocellular carcinoma according to aetiology. Best Pract Res Clin Gastroenterol. 2014; 28:937-47. doi: 10.1016/j.bpg.2014.08.006.

43. Mercer KE, Hennings L, Ronis MJ. Alcohol consumption, Wnt/beta-catenin signaling, and hepatocarcinogenesis. Adv Exp Med Biol. 2015; 815:185-95. doi: 10.1007/978-3-31909614-8_11.

44. Barneo L, Esteban MM, Garcia-Pravia C, Diaz F, Marin B. Normalization of the altered liver function tests after islet transplantation in diabetic rats. Diabete Metab. 1990; 16:284-9.

45. Patche J, Girard D, Catan A, Boyer F, Dobi A, Planesse C, Diotel N, Guerin-Dubourg A, Baret P, Bravo SB, ParadelaDobarro B, Alvarez E, Essop MF, et al. Diabetes-induced hepatic oxidative stress: a new pathogenic role for glycated albumin. Free Radic Biol Med. 2017; 102:133-48. doi: 10.1016/j.freeradbiomed.2016.11.026.

46. Ledda C, Loreto C, Zammit C, Marconi A, Fago L, Matera S, Costanzo V, Fuccio Sanza G, Palmucci S, Ferrante M, Costa C, Fenga C, Biondi A, et al. Noninfective occupational risk factors for hepatocellular carcinoma: A 
review (Review). Mol Med Rep. 2017; 15:511-33. doi: 10.3892/mmr.2016.6046.

47. Kariz S, Petrovic D. Interleukin-18 Promoter Gene Polymorphisms are not Associated with Myocardial Infarction in Type 2 Diabetes in Slovenia. Balkan J Med Genet. 2011; 14:3-9. doi: 10.2478/v10034-011-0011-6.

48. Dai ZJ, Liu XH, Kang HF, Wang XJ, Jin TB, Zhang SQ, Feng T, Ma XB, Wang M, Feng YJ, Liu K, Xu P, Guan HT. Genetic Variation in Metastasis-Associated in Colon Cancer-1 and the Risk of Breast Cancer Among the Chinese
Han Population: A STROBE-Compliant Observational Study. Medicine (Baltimore). 2016; 95:e2801. doi: 10.1097/ md.0000000000002801.

49. Wang Z, Liu X, Wang X, Chong T, Lin S, Wang M, Ma X, Liu K, Xu P, Feng Y, Dai Z. Polymorphisms in TIM3 and breast cancer susceptibility in chinese women: A case-control study. Oncotarget. 2016; 7:43703-43712. doi: 10.18632/oncotarget.9665. 